\title{
A First Attempt at Introducing Problem-Based Learning in an Engineering Dynamics Course
}

\author{
Tariq A. Khraishi \\ Mechanical Engineering Department \\ The University of New Mexico
}

\begin{abstract}
The engineering education literature, and other literature, is rich with references to the importance of Problem-Based Learning (PBL) in enhancing the educational experience of undergraduate students. In particular, PBL is supposed to strengthen concept learning in students. Such belief motivated the author to experiment with PBL in a required Dynamics class in the mechanical engineering (ME) department at the University of New Mexico (UNM). Due to the overall size of UNM and its mission, such a class typically involves a relatively large number of students (more than 40 typically). Such an enrollment figure encouraged the PBL experiment further since more interaction among students, or student groups, is probable and at least possible. The author found out that the most difficult part in the PBL experiment was picking one. The other thing found was that an extensive amount of effort went into making the experiment a successful one. The PBL experiment consisted of asking student groups to design an apparatus of some sort that is capable of shooting a golf ball in the air such that it lands in the middle of a sand-filled box at least one meter away. It was vital for the students to build their designs based on concepts learned in their Dynamics class and to explain, by equations, how their design works. The problem statement, as can be inferred, was open-ended and generated a spectrum of response from the students, i.e. different designs of the apparatus. A demonstration of all designs was held at the end of the semester. Although the students' learning, at least some of them, was enhanced by this experiment, the results did not prove to be as optimistic as initially hoped. Many students made major assumptions, namely neglecting friction in the workenergy equation, without verifying that such an assumption was a good or bad one. Indeed, this assumption turned out to be a bad one for some of the designs. Other problems arose when some groups tried to use a spring mechanism to initiate ball flight. This and other issues like what lessons were learned from this experiment are discussed in the paper.
\end{abstract}

\section{Introduction}

In the engineering education literature, Problem-Based Learning (PBL) is generally considered the best way to achieve the educational learning objectives as set forth in a Bloom's taxonomy. There has been a lot of work in the literature citing the benefits of PBL in enhancing student learning and discussing the issues associated with its implementation ${ }^{1,2,3,4}$. 
In the field of mechanics education, which tend to offer relatively hard classes such as statics, dynamics, and strength/mechanics of materials. PBL is not the only method to enhance students' learning. Indeed, educators have experimented with novel approaches (mostly computer-based) to try to do just that ${ }^{5,6,7,8}$. However, as mentioned above, PBL is generally considered a more rigorous approach that is proven to enforce concept learning in students. In mechanics courses, this is usually done by assigning design project(s) to groups of students ${ }^{9,10}$. Such design projects are by definition open-ended and have no unique answer or solution. The students thus have to invoke their imagination and try to integrate a host of previous classes (i.e. previous knowledge) in order to solve the problem at hand. This form of learning is considered the ultimate form since it is a replica of real engineering practice. It is worth noting that PBL is already existent in many engineering curricula, most notably through senior design courses. It is not common, however, in introductory engineering classes which are typically lecture-oriented. This paper discusses attempts at introducing PBL in a required sophomore/junior-level Dynamics course partly to get the students into the habit of building designs early on in their engineering education. Such attempts are complementary to departmental efforts of incorporating a five-course, four-year design sequence in its curriculum with the hope of graduating better engineers.

\section{The PBL Experiment}

The PBL experiment was conceptually simple. It consisted of asking student groups to design an apparatus of some sort that is capable of throwing a golf ball in the air for a horizontal distance of at least one meter such that it lands in the middle of a box topped-off with sand. The dimensions of the box were known apriori to the students. A golf ball on the other hand was, more or less, standard in its making and students had access to the machine shop and other labs to do any measurements (e.g. length or mass) they wanted. As it stood, the problem statement was rather open-ended and was thus expected to generate different apparatus designs. There was, however, a constraint on the design. It had to be explained by equations and principles learned during class and during class only. In other words, if some students came up with a mechanism that worked but they did not understand how its mechanics worked, then it would not have been accepted from them.

In response to the assigned projects, students assembled on their own in nominal groups of three (without any intervention from the instructor for the purpose of influencing the groups' composition). A total of 17 groups were formed. A variety of designs were built by the students all of which had to be demonstrated during one day at the end of the semester. Digital images of the apparatus at work were taken on that day with a portable digital camera and some are shown in this paper. Some of the designs involved a sliding mechanism whereby the golf ball had to slide down a curve or path of some sort to generate linear momentum capable of flying the ball for a horizontal distance of at least one meter (Figures 1 and 2 are examples). These designs relied on the fact that potential energy converted to kinetic energy during the downward slide of the ball. Other designs involved the use of a push or pull springs to drive the motion of the ball by converting the spring's potential energy into kinetic energy of the ball (Figure 3 is an example). One design used torsional and not linear springs (Figure 4). Several other designs employed some sort of an impact mechanism to drive the ball's motion (see Figure 5 for one example). Another design concept employed a catapult-type construction and is not shown here

Proceedings of the 2003 ASEE Gulf-Southwest Annual Conference

The University of Texas at Arlington

Copyright (c) 2003, American Society for Engineering Education 
for brevity. The last design concept used a straight tube elevated off the ground and sloped downwards (Figure 6).

\section{Experiment Results and Discussion}

The design project generated a lot of interest and enthusiasm amongst the students and an implied enhancement of students' grasping of course concepts was perceived. The results though are viewed as a first step only towards fine-tuning this PBL experiment for future reintroduction. For some students, the experiment still did not produce desired results. To illuminate, all of the groups that used a slide mechanism elected to ignore the effects of friction between the sliding ball and the rail or slide. The work-energy equation as was stated to them is: $U_{1-2}=T_{2}-T_{1}=\Delta T$

This equation simply states that the work done on a particle (left term) as it traverses a path between two points 1 and 2 is equal to the change in the kinetic energy $(\Delta T)$ of the particle between these two points. Here, although it was emphasized to the students in class (on several occasions indeed) that the left term accounts for all forces acting on the particle including frictional forces, all slide projects elected to neglect frictional effects without any justification to the appropriateness of such an assumption. As it turned out, neglecting friction resulted, in most cases, in a miscalculation of the off-floor release height by approximately a factor of two. The instructor has also built a slide mechanism to demonstrate to students during the exhibition day (shown on the left side of Figure 1). It was a relatively easy task to find the coefficient of friction between the ball and the slide material by timing the distance traveled by the ball on a straight portion of the slide material and back calculating from that the coefficient of kinetic friction. The probable causes why students elected to neglect friction are: 1) they were not sure how to calculate it and probably thought it will be a time-consuming endeavor, 2) they see that neglecting friction is a common assumption in many problems in standard Dynamics textbooks.

As explained earlier, some student groups built a spring-type mechanism to propel the golf ball. For those students who utilized a push-type (i.e. compression) spring, a selection that sounds more or less expected or natural for the application at hand, they did not realize three things: 1) it is very hard to determine a spring constant for this type of springs, 2) it takes a lot of effort to compress the spring in the first place, especially if you are using your hands for this purpose, 3 ) when releasing the compressed spring, it will spring back or elongate rapidly to its original free length. If you are using your hands, for example, your hands can then be in the way or interfere with the extension of the spring imparting thus less momentum onto the ball. Indeed, this unfortunate circumstance created serious problems for some of the groups utilizing compression springs in their designs.

For the groups that selected an impact-type mechanism to put the ball in flight, they also ran into some serious difficulties that apparently arose, in part, from weak understanding of the mechanics of impact problems. Some of these difficulties were: 1) the design of such apparatus is generally much more difficult than the above two mentioned mechanisms, 2) If the impact is not very well controlled it can result in either "direct central impact" or "oblique central impact" both of which result in different motion after the impact, 3) the students had no good understanding of how to calculate or estimate the "coefficient of restitution", a prime quantity in analyzing impact problems. Not only did they not know how to set-up a simple experiment that 
would have helped them estimate this coefficient (something that they were not taught in class), but they also did not realize that the value of this coefficient depends on the shape/geometry and material type of the impacting bodies and on their relative velocities at impact (although this part was mentioned to them in class).

Lastly, the one group that built the straight-pipe shoot performed very well in the project demonstration compared to other groups. Such a design easily allows for building a sturdy fixture. It also allows for repetitive and precise ball hits in the middle of the sand box, i.e. excellent target hits. For the project at hand, this design was the most simple and one of the most effective. Only one group out of 17 , however, came up with such a design.

\section{Lessons Learned and Conclusions}

There were quite a few lessons learned by the instructor from this PBL experiment:

1) The hardest part about the experiment was, in the first place, picking a good PBL problem that was relevant to the class material at hand. Some of the criteria that the author used in selecting a problem were: a) the design has to emphasize, or at least force the use of, concepts and equations learned in the classroom, b) the possible solution designs should be relatively simple to make or build, not costly in dollar amount, and not very time consuming.

2) The second thing learned was that the implementation of PBL takes a significant portion of the instructor's time.

3) The instructor needs to alert students to verify assumptions made in their design, or when they solve problems, before invoking such assumptions.

4) The student groups should be required to meet with the instructor at least once and sufficiently enough before the project deadline. The purpose of the meeting should be to discuss the design idea that they settled on in order to bring to their attention possible issues with their design and encourage their thought process on how to solve such perceivable problems. This is in an effort to circumvent the previously discussed problems or shortcomings faced by other student groups in the past.

5) The last thing learned is the need to better assess how PBL impacts student learning. For example, it should be possible to quiz students on knowledge before and after completing a PBL project and to try to statistically contrast the results. Such statistics would be the ever more enhanced if collected over the course of several semesters of teaching.

In conclusion thus, the author's first experiment with PBL in an engineering Dynamics class proved helpful although certainly not perfect. It appears that more preparation and careful planning for such experiments is an absolute must. Qualitative evidence pointed to the benefit of such an experiment to student learning as reflected by student feedback and general student enthusiasm about the assigned design project.

\section{References}

1. National Research Council, 2000, "How People Learn: Brain, Mind, Experience and School,” National Academy Press, Washington, D.C.

2. Woods, D. R., 1994, "Problem-Based Learning: How to Gain the Most from PBL," Publisher: Donald R. Woods, Waterdown, ON.

Proceedings of the 2003 ASEE Gulf-Southwest Annual Conference

The University of Texas at Arlington

Copyright (C) 2003, American Society for Engineering Education 
3. Edens, K., 2000, "Preparing Problem Solvers for the $21^{\text {st }}$ Century Through Problem-Based Learning," College Teaching, Vol. 48, No. 2, pp. 55-60.

4. Major, C. H., Palmer, B., 2001, "Assessing the Effectiveness of Problem-Based Learning in Higher Education: Lessons from the Literature," Academic Exchange Quarterly, Vol. 5, No. 1, pp. 4-9.

5. Ward, R. L., 1994, "Mastery Quizzes as a Teaching Tool in the Mechanics Series," Journal of Engineering Education, Vol. 83, No. 3, pp. 255-258.

6. Howell, K. C., 1996, "Introducing Cooperative Learning into a Dynamics Lecture Class," Journal of Engineering Education, Vol. 85, No. 1, pp. 69-72.

7. Flori, R. E., Koen, M. A., Oglesby, D. B., 1996, "Basic Engineering Software for Teaching ("BEST") Dynamics," Journal of Engineering Education, Vol. 85, No. 1, pp. 61-67.

8. Jacquot, R. G., Smith, D. A., Whitman, D. L., 1995, "Software Package to Enhance the Teaching of Engineering Dynamics," Computer Applications in Engineering Education, Vol. 3, No. 1, pp. 21-28.

9. Miller, G. R., Cooper, S. C., 1995, "Something Old, Something New: Integrating Engineering Practice into the Teaching of Engineering Mechanics," Journal of Engineering Education, Vol. 84, No. 2, pp. 105-115.

10. Thompson, B. E., 2002, "Pedagogy of an Aircraft Studio," Journal of Engineering Education, Vol. 91, No. 2, pp. 197-201.

TARIQ A. KHRAISHI

Dr. Khraishi currently serves as an Assistant Professor of Mechanical Engineering at the University of New Mexico. His general research interests are in theoretical, computational and experimental solid mechanics and materials science. He has taught classes in Dynamics, Materials Science, Advanced Mechanics of Materials, Elasticity and Numerical Methods. For the last two-three years he has engaged himself in the scholarship of teaching and learning. 


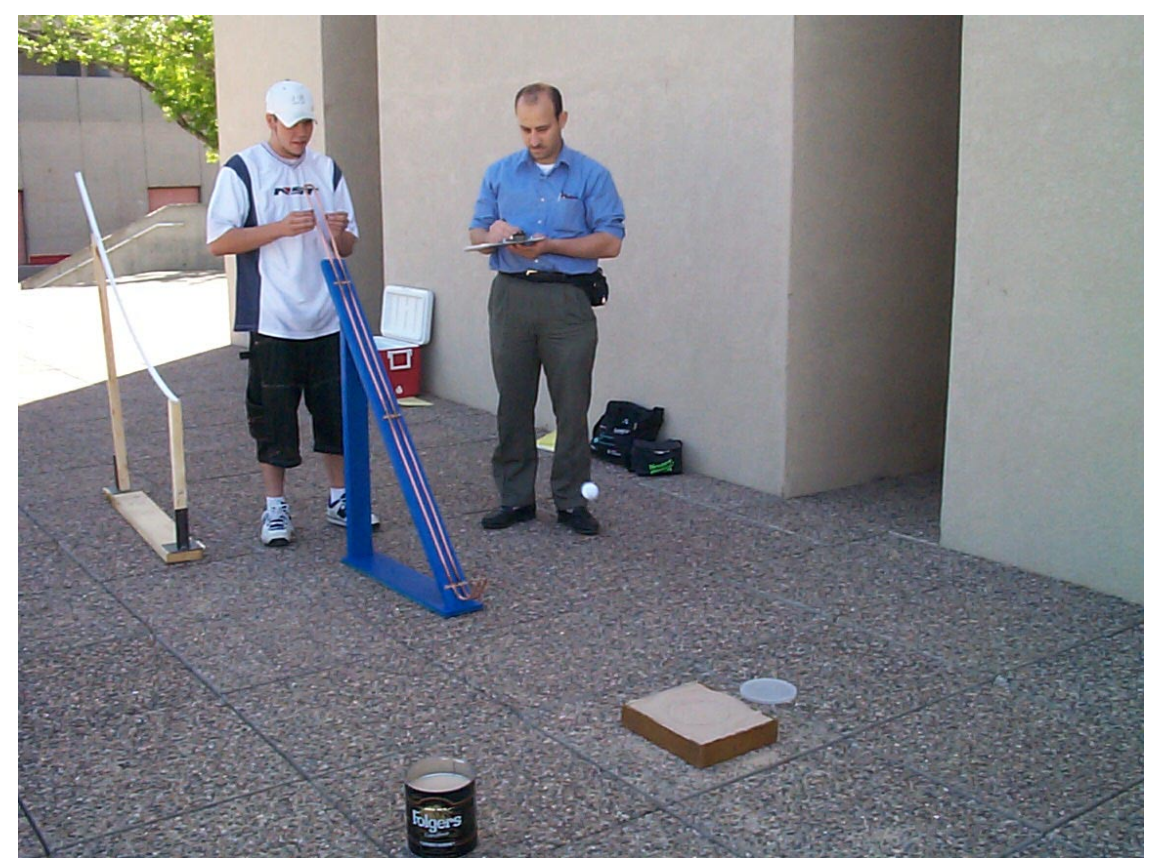

Figure 1. One of the slide designs used to land the golf ball in the sand-filled box one meter away. Notice the white golf ball in the air in this figure and others.

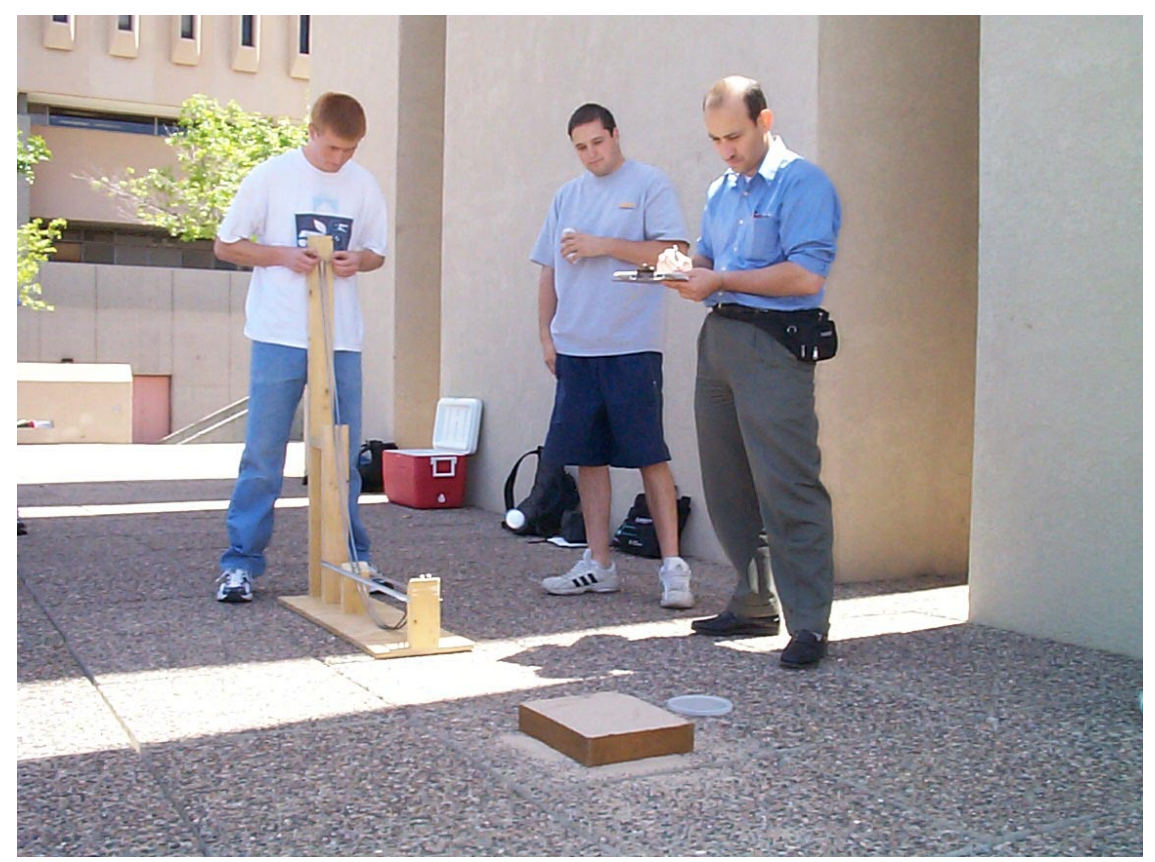

Figure 2. Another slide design that released a golf ball from a certain height off the ground to gain linear momentum. 


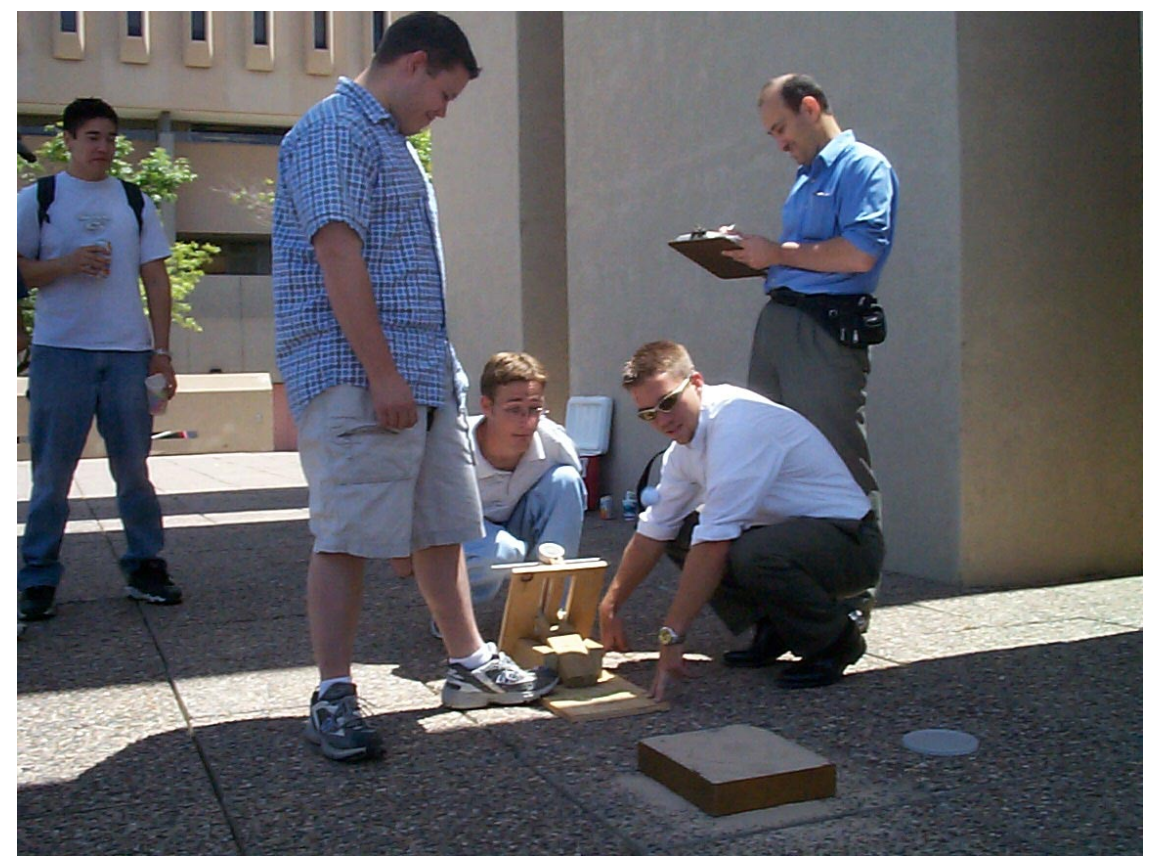

Figure 3. A design utilizing a compression spring tilted at an angle from the floor.

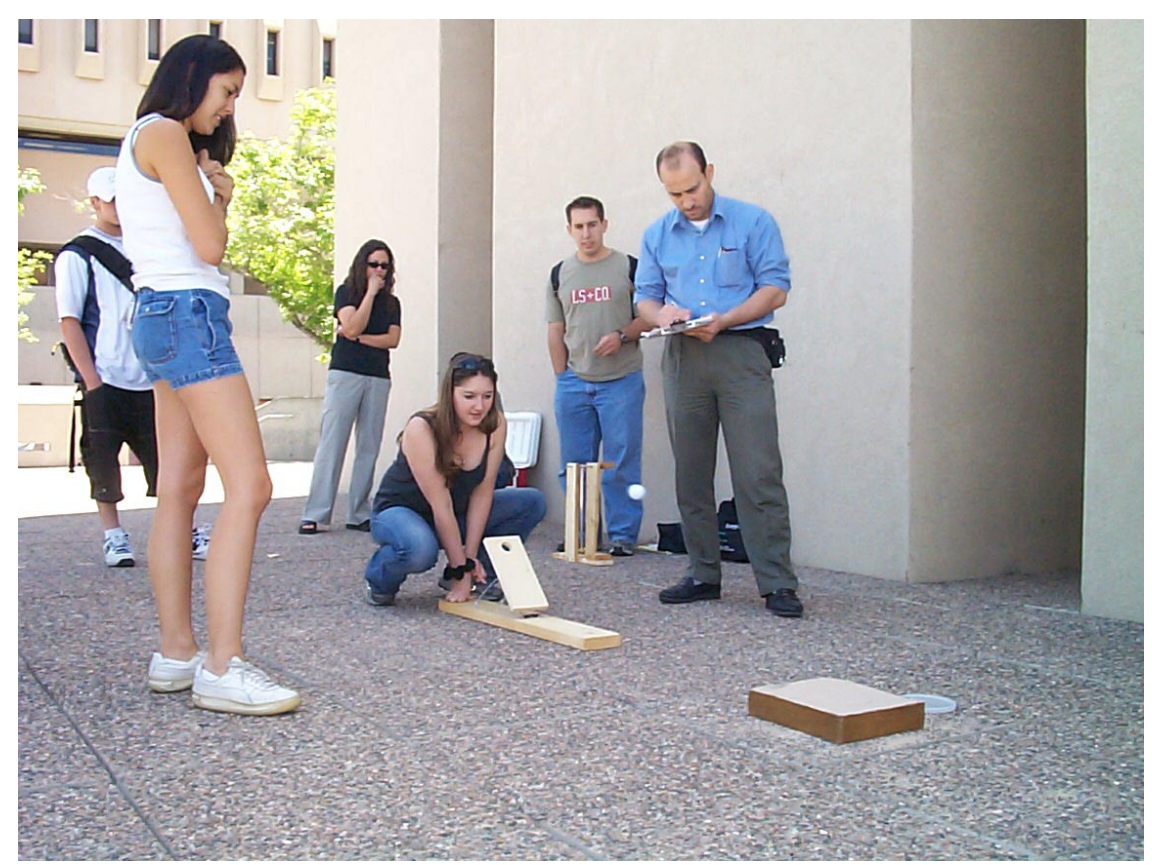

Figure 4. A torsional spring design where the ball rested loosely in a smaller hole in a springing wood board. 


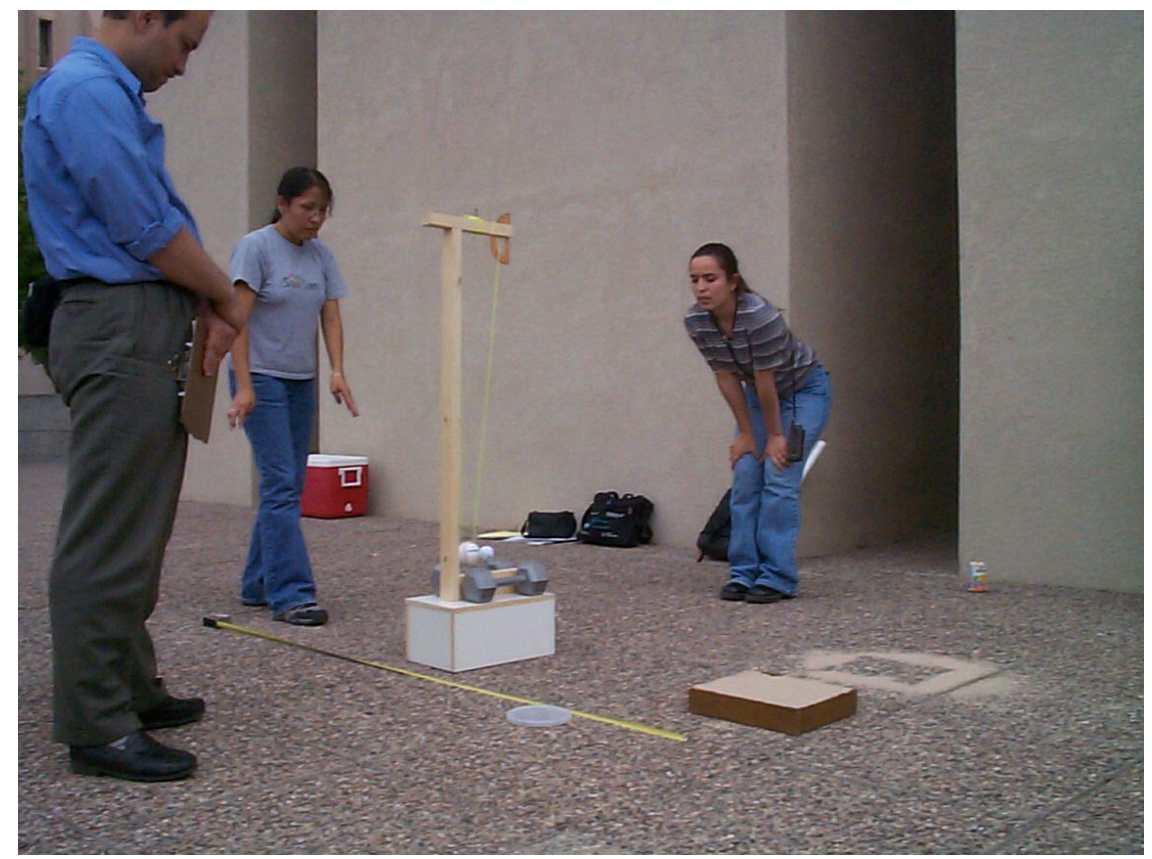

Figure 5. A design that relied on the impact of a pendulum with the golf ball in order to shoot the ball in the air. In this picture, the pendulum (composed of a baseball attached to strings) is showing as it was just about to hit the golf ball.

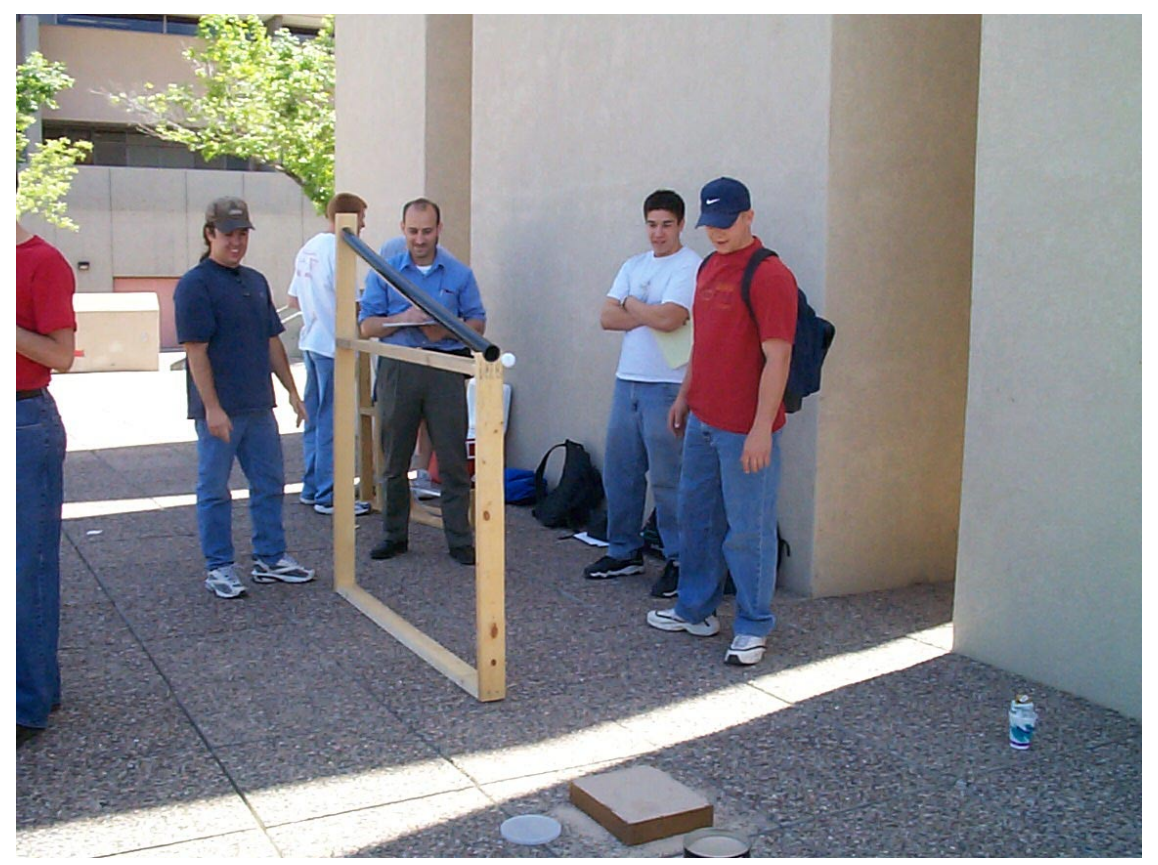

Figure 6. A design with a straight-pipe shoot. 